\title{
Study of Predisposing Factors and Management of Carcinoma of the Tongue
}

\author{
M. Natesan ${ }^{1}$, M. Raja ${ }^{1}$ \\ ${ }^{1}$ Assistant Professor, Department of General Surgery, Government Medical College, Pudukkottai, Tamil Nadu, India
}

Corresponding author: Dr. M. Raja, Assistant Professor, Department of General Surgery, Government Medical College, Pudukkottai, Tamil Nadu, India

DOI: http://dx.doi.org/10.21276/ijcmsr.2019.4.3.47

How to cite this article: M. Natesan, M. Raja. Study of predisposing factors and management of carcinoma of the tongue. International Journal of Contemporary Medicine Surgery and Radiology. 2019;4(3):C217-C219.

\section{A B S T R A C T}

Introduction: Oral cancer is particularly dangerous because in its early stages it may not be noticed by the patient, as it can frequently prosper without producing pain or symptoms they might readily recognize, and because it has a high risk of producing second, primary tumors. Current research aimed to study various predisposing factors, age, sex distribution of carcinoma of the tongue.

Material and Methods: 100 patients were included in this study. Data collected from patient records included patient and tumor characteristics and pretreatment staging, reported tobacco and alcohol use, treatment modalities, and outcome.

Results: In our study roughly $73 \%$ of patients belonged to stage IV disease. According to the sex ratio Male is about $84 \%$ and females are $16 \% .65 \%$ of the patients were smokers, $35 \%$ of the patients were tobacco chewers and betal nut chewier. $75 \%$ of the patients presented with cervical nodal secondaries. FNAC was done in $92 \%$ of the nodal positive cases. More than $50 \%$ of the patients were subjected to surgical management. Recurrence was very much minimal with surgery followed by postoperative radiotherapy which was $9 \%$.

Conclusion: The importance of early detection for clinical downstaging is stressed. Any ulcer or lesion at a younger age should not be dismissed easily, even if it is not habit related

Keywords: Oral Cancer, Squamous Cell, Risk Factors

\section{INTRODUCTION}

The tongue is a vital structure essential for initiating the first phase of swallowing, moving food in the oral cavity to permit adequate mastication to enjoy the taste of delicious food. Early detection is the key to an effective cure of carcinoma tongue with minimal disability. The tongue is a highly muscular organ of deglutition, taste and speech. Partly oral and partly pharyngeal in position and is attached by its muscles to the hyoid bone, mandible, styloid processes, soft palate and the pharyngeal wall. It has a root, apex, a curved dorsum and an inferior surface. The mucosa is normally pink, moist and attached closely to the underlying muscles. The root of the tongue is attached to the hyoid bone and mandible. The dorsum is divided by $\mathrm{V}$ Shaped sulcus terminalis into an anterior, oral or presulcal part which is facing upwards; Posterior, Pharyngeal or postsulcal part facing posteriorly which forms posterior $1 / 3$ of the tongue. The two limits of sulcus terminalis run anterolaterally to the palatoglossal arches from a median depression - foramen caecum which is the site of upper end of the embryonic thyroid diverticulum. ${ }^{1}$ Tobacco appears to be a risk factor for most cancers originating in the tongue. Studies have demonstrated an increased risk of oral, pharyngeal, laryngeal cancer among alcoholics. $^{2-4}$ Trauma and local irritation are considered extremely important in the aetiology of carcinoma tongue.
Malocclusion producing chronic cheek bite, ill-fitting dentures, sharp teeth which constantly irritate the mucosa. It is well recognized that deficiency of Vitamin A will induce metaplasia and keratinization of certain epithelial structures. Vitamin B complex deficiency has also been suggested as a predisposing factor which must be related to the alteration in the oxidation patterns of the epithelium.,

Current research aimed to study various predisposing factors, age, sex distribution of carcinoma of the tongue.

\section{MATERIAL AND METHODS}

Totally 100 patients were chosen from the outpatient department of surgery from January 2014 to January 2017 in government head quarters Hospital, pudukkottai. Inclusion criteria: Patients having ulceroproliferative growth over the tongue. Biopsy confirming the malignant nature of the disease or FNAC from the neck node shows positive for malignant cells. The age, sex, and anatomical site of all cases were compiled from the clinical data sent together with the biopsy records.

Data collected from patient records included patient and tumor characteristics and pretreatment staging, reported tobacco and alcohol use, treatment modalities, and outcome. The histopathologic diagnosis and report of mild, moderate, or poorly differentiated SCC were graded according to World Health Organization guidelines. 


\section{RESULTS}

Majority of the patient attended our outpatient department were at a very late stage. In our study roughly $73 \%$ of patients belonged to stage IV disease and only around $18 \%$ were stage I and II. Usually patients reported for ulcer over the tongue and only, the patients visited the hospitals. Invariably all the patients were having increased salivation. Patients with Carcinoma of the posterior $1 / 3$ of the tongue, usually did not have any symptom at an early stage. In posterior $1 / 3$ cancers, the patients visited for pain over the throat or swelling in the neck usually at the later stage and also have dysphagia.

Almost all the patients were with poor oral and dental hygiene. According to the sex ratio Males was about $84 \%$ and females were $16 \%$ (table-1).

Roughly $70 \%$ of the patients were more than 50 years and only $4 \%$ of patients were less than 30 years (table-2).

$65 \%$ of the patients were smokers. Usually $10-15$ beedies per day for more than 20-30 years. All the developed usually the pain (table-3).

Roughly $35 \%$ of the patients were tobacco chewers and betal nut chewier. Majority of the patients keep them inside the oral cavity for long time even in the night (table-4).

Caries teeth presented in $48 \%$ of the patients. Premalignant lesions like leukoplakia were present around 13\% of the patients and submucous fibrosis presented in 3\% of the patients. Sharp teeth presented in about $49 \%$ of the patients, especially near the site of the ulcer or growth of the tongue. No sharp teeth presented in $51 \%$ of the patients. This was due to loss of teeth for treatment purpose, caries teeth and poor dental hygiene.

Male patients especially from the rural area were invariably alcoholics. Alcoholics roughly were about $55 \%$ of the patients (table-5).

When we examined the patients right lateral border of the tongue was the usual site of carcinoma of the tongue. Involvement of right lateral border was $45 \%$ only two patients had lesions at the tip of the tongue.

$75 \%$ of the patients presented with cervical nodal secondaries. Unilateral involvement of nodes were around 55\% and bilateral involvement was $41 \%$ of the patients. Contralateral nodes were involved in $4 \%$ of the patients.

FNAC was done in $92 \%$ of the nodal positive cases. We did not attempt in very small nodes. The FNAC gave $72 \%$ positive result for nodal metastasis. Negative in $28 \%$ of patients. It might be due to nodes per Se nonmetastatic or technically not reached the metastatic sites. So FNAC is the ideal method to find out nodal metastasis. More than 50\% of the patients were subjected to surgical management. 2 patients were having small ulcer over the tip of the tongue and were treated with excision of the ulcer and primary closure of the defect. Hemiglossectomy is about $42 \%$ of the patients. Surgery was done after primary radiotherapy in about $16 \%$ of the patients.

In the surgical patient's block dissection done in $73 \%$ of the patients. $68 \%$ of the block dissection were unilateral. Nodes in the block dissection specimen were positive for metastasis in $74 \%$. Radiotherapy was given to $68 \%$ of the patients under study. In which $70 \%$ of the patients were treated primarily with radiotherapy. Only $30 \%$ of the patients received

\begin{tabular}{|l|c|}
\hline Stage of the disease & Percentage of the disease \\
\hline I & $8 \%$ \\
\hline II & $10 \%$ \\
\hline III & $9 \%$ \\
\hline IV & $73 \%$ \\
\hline \multicolumn{2}{|c|}{ Table-1: Distribution of Stages } \\
\hline
\end{tabular}

\begin{tabular}{|l|c|}
\hline Sex & Percentage of distribution \\
\hline Male & $84 \%$ \\
\hline Female & $16 \%$ \\
\hline \multicolumn{2}{|c|}{ Table-2: Distribution of gender } \\
\hline
\end{tabular}

\begin{tabular}{|l|c|}
\hline Age & Percentage of distribution \\
\hline $0-10$ years & 0 \\
\hline $10-20$ years & 0 \\
\hline $20-30$ years & $4 \%$ \\
\hline $30-40$ years & $10 \%$ \\
\hline $40-50$ years & $16 \%$ \\
\hline $50-60$ years & $30 \%$ \\
\hline $60+$ years & $40 \%$ \\
\hline \multicolumn{2}{|c|}{ Table-3: Distribution of Age group } \\
\hline
\end{tabular}

\begin{tabular}{|l|c|}
\hline Smoking status & Percentage of distribution \\
\hline Smokers & $65 \%$ \\
\hline Non smokers & $35 \%$ \\
\hline \multicolumn{2}{|c|}{ Table-4: Distribution of smokers and non-smokers } \\
\hline
\end{tabular}

\begin{tabular}{|l|c|}
\hline Risk factors & Percentage of distribution \\
\hline Tobacco chewing & $35 \%$ \\
\hline Caries tooth & $48 \%$ \\
\hline Alcoholism & $55 \%$ \\
\hline \multicolumn{2}{|c|}{ Table-5: Distribution of risk factors } \\
\hline
\end{tabular}

postoperative radiotherapy. Neoadjuvant chemotherapy was given to no patients. Adjuvant chemotherapy along with radiotherapy was given to $16 \%$ and palliative chemotherapy given to the massive diseases was $12 \%$ of the patients. The drugs we used were cisplatin, methotrexate and 5FU. Recurrence rate in our study was $28 \%$. After surgery alone the recurrence was $23 \%$ and after radiotherapy alone $42 \%$. Recurrence was very much minimal with surgery followed by postoperative radiotherapy which was $9 \%$. The biopsy report for all the patients came as squamous cell carcinoma except two patients, in which the reports were nonspecific ulcers. No patient had the reconstructive procedure after surgical management of carcinoma of the tongue.

\section{DISCUSSION}

Oropharyngeal cancer (OPC) accounts for $2 \%$ of all body cancer in the United States and approximately 50\% of the patients die of their disease. Similar statistics are demonstrated for the Canadian population where $1.6 \%$ of all estimated deaths attributed to cancer in 2003 are those of oropharyngeal cancers. ${ }^{7}$ In general, the incidence of OPC in males is twice that in women and occurs mainly in the fifth and sixth decade of life. ${ }^{8}$ 
The preponderance of male cases as compared to female cases (1.5:1 ratio) and the mean age (61 years) were consistent with other oropharyngeal cancer demographic reports in general and of earlier reports of tongue cancer. ${ }^{9}$ Ten percent of our patients were younger than 40 years at the time of diagnosis, which is much greater than the $1 \%$ to $3 \%$ that was reported for oropharyngeal cancer in general, but this increased proportion of oral cancer and tongue cancer in young patients may be part of a general trend of oral cancer incidence in younger individuals. ${ }^{10}$ It was also reported that the incidence of oropharyngeal cancer in less than a decade increased in white males ages 15 to 34 years by $267 \% .^{11}$

The majority of patients in this study had a history of tobacco and alcohol use. The increased risk of oral cancer in tobacco and alcohol consumers is well known. ${ }^{12}$ It is believed that smoking is directly attributable to about $92 \%$ of oral cavity tumors in men and $61 \%$ of those in women, as well as to the high risk of developing a second primary cancer. ${ }^{13}$ The risk of developing oral cancer in smokers also increases with the number of cigarettes smoked per day. Alcohol is considered a promoter and a possible cocarcinogen to tobacco and contains congeners and carcinogenic contaminants. ${ }^{14}$ Excess risk of approximately 10 -fold is associated with a combination of tobacco and alcohol use. ${ }^{15}$ Oropharyngeal cancers are rare in people who do not smoke, however nonsmokers who consume alcohol have a 3-fold higher risk of developing oropharyngeal cancer compared to nonsmokers and nondrinkers. ${ }^{16}$

The treatment focus for SCC of the oral tongue included primarily surgical excision, primarily radiotherapies, or a combination of both radiotherapy and surgery. ${ }^{17}$ In a similar fashion the treatment for SCC of the base of the tongue also varied from primarily surgery to radiation and surgery. ${ }^{18}$

\section{CONCLUSION}

Clinicians should be aware of the high-risk groups of SCC of the tongue and its common presenting symptoms. A thorough head and neck and oral examination are necessary. While changes are occurring in the approach to the treatment of advanced stage tongue cancer including altered radiation therapy, intensity-modulated radiation therapy, and combinations with chemotherapy, these changes may affect survival and quality of life.

\section{REFERENCES}

1. Review Gray's Anatomy: The Anatomical Basis of Clinical Practice / 41th edition. - Elsevier, 2015. - 1584 p. (2015). SI 'DMA'.

2. Fernandez G L, Sankaranarayanan R, Anta J J Lence, Rodriguez S A, Parkin D M.1995. An Evaluation of the Oral Cancer Control Program in Cuba. Epidemiology 6(4): 428-31.

3. Lambert $\mathrm{R}$, Sauvaget C, Camargo C M De, Sankaranarayanan R. Epidemiology of Cancer from the Oral Cavity and Oropharynx. European Journal of Gastroenterology \& Hepatology 2011; 23 (8): 633-41.

4. Gupta P C, Ray C S, Sinha D N, Singh P K. Smokeless Tobacco: A Major Public Health Problem in the SEA Region: A Review." Indian Journal of Public Health 2011; 55 (3): 199-209.

5. Lucenteforte E, Garavello W, Bosetti C, La V C.
Dietary Factors and Oral and Pharyngeal Cancer Risk. Oral Oncology 2009; 45(6): 461-67.

6. World Cancer Research Fund and AICR (American Institute for Cancer Research). 2007. Food, Nutrition, Physical Activity, and the Prevention of Cancer: A Global Perspective. Washington, DC: AICR.

7. Landis SH, Murray T, Bolden S, Wingo PA. Cancer statistics. CA Cancer J Clin 1998;48(2):6-29.

8. Shaha AR, Strong EW. Cancer of the head and neck. In: Murphy GP, Lawrence W Jr, Lenhard RE Jr, editors. American Cancer Society textbook of clinical oncology. Washington, DC: American Cancer Society; 1995. p. 355-77.

9. Callery CD, Spiro RH, Strong EW. Changing trends in the management of squamous carcinoma of the tongue. Am J Surgery 1984;148(2):449-54.

10. Shiboski CH, Silverman S Jr. Oral Cancer epidemiology update: analysis of surveillance epidemiology and end results data, 1973- 1999. Oral Surg Oral Med Oral Pathol Oral Radiol Endod 2003; 95(3):422.

11. Devesa SS, Blot WJ, Stone BJ, Miller BA, Tarone RE, Fraumeni JF. Recent cancer trends in the United States. J Natl Cancer Inst 1995;87(6):175-82.

12. Spitz MR. Epidemiology and risk factor for head and neck cancer. Sem Oncol 1994;21(1):281-8.

13. Silverman S Jr, Gorsky M, Greenspan D. Tobacco usage in patients with head and neck carcinomas: a followup study on habit changes and second primary oral/ oropharyngeal cancers.J Am Dent Assoc 1983;106(4):335.

14. Weisburger JH, Williams GM. Causes of cancer. In: Murphy GP, Lawrence W Jr, Lenhard RE Jr, editors. American Cancer Society textbook of clinical oncology. Washington, DC: American Cancer Society; 1995. p. 10-39.

15. Blot WJ, McLaughlin JK, Winn DM, Austin DF, Greenberg RS, Preston-Martin S, et al. Smoking and drinking in relation to oral and pharyngeal cancer. Cancer Res 1988;48(3):3282-7.

16. Fioretti F, Bosetti C, Tavani A, Franceschi S, La Vecchia C. Risk factors for oral and pharyngeal cancer in never smokers. Eur J Cancer Oral Oncol 1999;35(3):375-8.

17. Nyman J, Mercke C, Linstrom J. Prognostic factors for local control and survival of cancer of the oral tongue. Acta Oncol 1993;32(3):667-73.

18. Harrison LB, Session RB, Strong EW, Fass ED, Nori D, Fuks A. Brachytherapy as part of the definitive management of squamous cancer of the base of tongue. Int J Radiat Oncol Biol Phys 1989; 17(2):1309-12.

\section{Source of Support: Nil; Conflict of Interest: None}

Submitted: 13-07-2019; Accepted: 13-08-2019; Published online: 21-09-2019 